\title{
Estudo fitoquímico e avaliação da atividade larvicida de Pterodon polygalaeflorus Benth (Leguminosae) sobre Aedes aegypti
}

\author{
Antonia T.A. Pimenta ${ }^{1}$, Gilvandete M.P. Santiago ${ }^{1,2 *}$, Angela M.C. Arriaga ${ }^{2}$, Gustavo H.A. \\ Menezes $^{2}$, Suzana B. Bezerra ${ }^{2}$
}

\author{
${ }^{1}$ Departamento de Química Orgânica e Inorgânica, Universidade Federal do Ceará, \\ CP 12200, 60021-970, Fortaleza, CE, Brasil, \\ ${ }^{2}$ Departamento de Farmácia, Faculdade de Farmácia, Odontologia e Enfermagem, \\ Universidade Federal do Ceará, 60430-370, Fortaleza, CE, Brasil
}

\begin{abstract}
RESUMO: Estudo fitoquímico dos extratos hexânico e metanólico dos frutos de Pterodon polygalaeflorus Benth forneceu três diterpenos furânicos: 6- $\alpha$-acetoxivouacapano, 6- $\alpha-$ hidroxivouacapano e vouacapano, sendo este ultimo relatado pela primeira vez como produto natural. Avaliação da atividade sobre larvas de estágio 3 de Aedes aegypti de 6- $\alpha$-acetoxivouacapano, dos extratos hexânico e metanólico dos frutos de P. polygalaeflorus e de seu óleo essencial também é relatada. Apenas o extrato hexânico mostrou boa atividade com $\mathrm{CL}_{50} 23,99 \pm 0,75 \mu \mathrm{g} / \mathrm{mL}$, podendo ser considerado um potencial agente larvicida.
\end{abstract}

Unitermos: Pterodon polygalaeflorus, Aedes aegypti, diterpenos furânicos, atividade larvicida.

\begin{abstract}
Phytotochemical study and evaluation of larvicidal activity of Pterodon polygalaeflorus Benth (Leguminosae) against Aedes aegypti". Chemical investigation of the hexane and methanol extracts of the fruits of Pterodon polygalaeflorus (Leguminosae) resulted in the isolation and identification of furanic diterpenes: 6- $\alpha$-acetoxivouacapane, $6-\alpha-$-hidroxyvouacapane and vouacapane, which is related by the first time as a natural product. The structures of these compounds were established by spectroscopic analysis, including 2D NMR experiments. The 6$\alpha$-acetoxivouacapane, the hexane and methanol extracts, and the essential oil were evaluated on $3^{\text {rd }}$ instar larvae of Aedes aegypti and only the hexane extract showed good larvicidal activity with $\mathrm{LC}_{50} 23.99 \pm 0.75 \mu \mathrm{g} / \mathrm{mL}$.
\end{abstract}

Keywords: Pterodon polygalaeflorus, Aedes aegypti, furanic diterpenoids, larvicidal activity.

\section{INTRODUÇÃO}

O táxon genérico Pterodon (Leguminosae) é constituído por cinco espécies distribuídas no Brasil: $P$. abruptus Vog., P. apparicioi Pedersoli, P. emarginatus Benth, P. polygalaeflorus Benth e P. pubescens Benth. Estudos relatam a ação profilática do óleo dos frutos de $P$. pubescens sobre Schistossoma mansoni em camundongos, sendo esta atividade atribuída ao 14,15diidroxigeranilgeraniol (Demuner et al., 1996) e, atividade antiinflamatória (Falcão et al., 2005). Os constituintes característicos deste táxon genérico são diterpenos lineares e tetracíclicos, apresentando esqueletos vinhaticano ou vouacapano (Campos et al., 1994a). P. polygalaeflorus é conhecida popularmente como sucupira, sucupirabranca, sucupira-lisa, faveiro-azul (Lorenzi,1998) e é usada na medicina popular contra bronquites, amigdalites e como tônico (Arriaga et al., 2000). Estudos sobre ação antimicrobiana e inibidor da enzima acetilcolinesterase encontram-se descritos na literatura (Lima et al., 2006 e Barbosa-Filho et al., 2006).
O mosquito Aedes aegypti é o agente transmissor da dengue, doença infecciosa causada por um arbovírus, endêmica no Brasil e transmitida de uma pessoa doente para outra sadia, pela picada da fêmea contaminada do mosquito. O desenvolvimento de resistência do vetor da dengue aos inseticidas químicos (Santiago et al., 2005) e sua toxicidade levam à busca de novos inseticidas naturais.

A busca de novos inseticidas e o crescente estímulo às pesquisas que visam o uso de plantas como uma alternativa para o controle de mosquitos, vetores de doenças, como a dengue, são motivados pela quase ausência de toxicidade destes produtos naturais a animais e plantas, e pelo fato deles serem biodegradáveis, o que evita a contaminação do meio ambiente. Em contraste, os inseticidas sintéticos, aos quais os insetos se tornam cada vez mais resistentes (Santiago et al., 2005), são tóxicos e poluentes.

Assim, o presente trabalho mostra o isolamento dos diterpenos furânicos dissubstituídos vouacapano (1), 6- $\alpha$-acetoxivouacapano (2) e 6- $\alpha$-hidroxivouacapano 
(3) (Figura1). As estruturas destes compostos foram elucidadas, principalmente, através da análise de seus dados espectroscópicos de $\mathrm{RMN}{ }^{1} \mathrm{H}$ e $\mathrm{RMN}{ }^{13} \mathrm{C}$ uni e bidimensionais. $\mathrm{O}$ composto $\mathbf{1}$ é relatado pela primeira vez como produto natural. As atividades larvicida dos extratos hexânico e metanólico, do composto 2 e do óleo essencial de $P$. polygalaeflorus são também descritas.

\section{MATERIAL E MÉTODOS}

\section{Métodos gerais}

Os espectros na região do infravermelho foram obtidos em espectrômetro Perkin-Elmer, modelo FTIR 1000. Foram utilizadas pastilhas de brometo de potássio. Os espectros de $\mathrm{RMN}{ }^{1} \mathrm{H}$ e $\mathrm{RMN}{ }^{13} \mathrm{C}$, uni e bidimensionais, foram registrados em espectrômetro Bruker, modelo DRX-300 e DPX-500. Os deslocamentos químicos $(\delta)$ foram expressos em partes por milhão (ppm) e foram referenciados para $\mathrm{RMN}{ }^{1} \mathrm{H}$ pelos picos referentes à fração não deuterada do clorofórmio $(\delta$ $7,27)$. Para $\mathrm{RMN}{ }^{13} \mathrm{C}$ pelos picos de $\mathrm{C}-13$ do clorofórmio $(\delta 77,0)$. A análise do óleo essencial obtido foi realizada em cromatógrafo gasoso Shimadzu, modelo CG-17A, provido de coluna capilar de metil-fenil silicone DB-5 com $25 \mathrm{~m}$ de comprimento, $0,20 \mathrm{~mm}$ de diâmetro interno acoplado a um espectrômetro de massas Shimadzu. O cromatograma com área detectada do óleo essencial foi obtido através de detector de ionização por chama, provido de coluna capilar de metil-fenil silicone DB5 com 30,0 $\mathrm{m}$ de comprimento e $0,20 \mathrm{~mm}$ de diâmetro interno. Para as colunas cromatográficas utilizou-se gel de sílica com granulometria de 0,023 a 0,200 mm. As análises cromatográficas em camada delgada foram efetuadas em gel de sílica G 60 sobre suporte de vidro e em gel de sílica $\mathrm{F}_{254}$ sobre poliéster. As placas cromatográficas foram reveladas através da irradiação com luz de ultravioleta em comprimentos de onda de 254 e $366 \mathrm{~nm}$ e/ou aquecimento após borrifação com uma solução de vanilina em ácido perclórico e etanol. Os valores de ponto de fusão das substâncias isoladas foram determinados em aparelho de microdeterminação digital da Mettler Toledo provido de uma estação de aquecimento FP82HT e uma central de processamento FP90. As determinações foram feitas a uma velocidade de $4{ }^{\circ} \mathrm{C}$ por minuto e não foram corrigidas. As análises de poder rotatório específico foram realizadas em um polarímetro 341 da Perkin Elmer.

\section{Material vegetal}

O material botânico foi adquirido no herbanário do mercado São Sebastião, localizado no município de Fortaleza. A exsicata da planta encontra-se arquivada no Herbário Prisco Bezerra da Universidade Federal do Ceará, sob o número 8808.

\section{Ensaio larvicida}

Nos bioensaios, os ovos de A. aegypti foram eclodidos em água isenta de cloro, à temperatura ambiente. Em condições normais, os ovos maduros eclodem quando submersos em meio líquido e apresentam quatro estágios larvários, sendo selecionadas larvas de estágio 3 (Gadelha; Toda, 1985) para serem utilizadas no presente estudo.

Em um béquer de $50 \mathrm{~mL}$, à temperatura ambiente, as amostras testadas, em diferentes concentrações $(12,5 \mu \mathrm{g} / \mathrm{mL}$ a $500 \mu \mathrm{g} / \mathrm{mL})$ foram dissolvidas em $0,3 \mathrm{~mL}$ de DMSO e a cada solução foram adicionadas 50 larvas de estágio 3, completandose o volume para $20 \mathrm{~mL}$ com água. Após 24 horas, à temperatura ambiente, as larvas mortas foram contadas e calculada a percentagem letal e posteriormente a $\mathrm{CL}_{50}$. Os testes foram feitos em triplicata (Oliveira et al., 2002). Paralelamente foram feitos testes em branco, utilizandose DMSO e água.

\section{Extração e isolamento dos constituintes químicos}

Para obtenção do óleo essencial, os frutos secos e triturados de $P$. polygalaeflorus Benth $(500 \mathrm{~g})$ foram submetidos à hidrodestilação em aparelho de Cleavenger modificado por 2 horas, sendo obtidos $1,9 \mathrm{~mL}$ do óleo essencial.

Para obtenção dos extratos os frutos $(1,5 \mathrm{~kg})$, triturados mecanicamente, foram submetidos à extração com hexano em aparelhagem Soxhlet. Após remoção do solvente em evaporador rotatório, obteve-se 364,47 $\mathrm{g}$ de extrato hexânico. A torta resultante foi submetida à extração exaustiva com metanol a quente, e forneceu 96,0 g de extrato metanólico.

O extrato hexânico $(50,0 \mathrm{~g})$ foi adsorvido em gel de sílica, pulverizado em gral de porcelana e submetido à cromatografia em coluna em gel de sílica, utilizando-se hexano, clorofórmio e acetato de etila como eluentes. A fração $1(1,46 \mathrm{~g})$ eluída com hexano foi recromatografada em coluna de gel de sílica $(60 \mathrm{~g})$, utilizando-se um gradiente de eluição crescente com hexano e diclorometano. Após análise por comatografia em camada delgada, a fração eluída com hexano $(0,137 \mathrm{~g})$ foi recromatografada em coluna de gel de sílica $(9,0 \mathrm{~g})$, utilizando-se hexano como eluente, forneceu $0,01 \mathrm{~g}$ de cristais brancos que foram identificados como vouacapano (1).

O extrato metanólico $(96,0 \mathrm{~g})$ foi fracionado por cromatografia em coluna de gel de sílica, usando como eluentes hexano, diclorometano, acetato de etila e metanol. A fração eluída com hexano: diclorometano (50:50) continha cristais amarelos que foram recristalizados em metanol e o sólido obtido $(181,0 \mathrm{mg})$ foi identificado como 6- $\alpha$-acetoxivouacapano (2). A fração 3 (3,8 g), eluída com diclorometano, foi submetida à cromatografia em coluna de gel de sílica $(60 \mathrm{~g})$ com gradiente de polaridade e eluição com hexano e acetato de etila. A fração eluída com hexano : acetato de etila (95 : 05$)$ originou material sólido $(1,0 \mathrm{~g})$ que foi purificado 
em fracionamento cromatográfico isocrático, utilizando hexano : diclorometano $(40: 60)$ como eluente e gel de sílica (71 g) como adsorvente, do qual obteve-se $15 \mathrm{mg}$ de 6- $\alpha$-hidroxivouacapano (3).

\section{RESULTADOS E DISCUSSÃO}

O fracionamento por cromatografia em coluna do extrato hexânico, levou ao isolamento e identificação do diterpeno furânico vouacapano (1), enquanto o tratamento cromatográfico do extrato metanólico em coluna de sílica forneceu dois diterpenos furânicos: 6$\alpha$-acetoxivouacapano (2) e 6- $\alpha$-hidroxivouacapano (3) (Figura 1).

O diterpeno 1, sólido branco amorfo, que apresentou ponto de fusão na faixa de $71,3-73,5^{\circ} \mathrm{C}$ e rotação específica $[\alpha]_{\mathrm{D}}{ }^{25}=-362,22\left(\mathrm{c} 1,8, \mathrm{CHCl}_{3}\right)$, mostrou em seu espectro de $\mathrm{RMN}{ }^{1} \mathrm{H}$ três singletos, em $\delta 0,88, \delta 0,91$ e $\delta 0,91$, correspondentes aos átomos de hidrogênio de grupos metila ligados a átomos de carbono não hidrogenado, um dubleto em $\delta 0,99(\mathrm{~J}=7,0 \mathrm{~Hz})$ correspondente aos átomos de hidrogênio de grupo metila ligado a carbono mono-hidrogenado. Mostrou ainda dois dubletos em $\delta 6,19(J=1,75 \mathrm{~Hz})$ e $\delta 7,23(J=1,75 \mathrm{~Hz})$, indicando a presença de um anel furânico dissubstituído, bastante encontrado no gênero Pterodon. Observou-se no espectro de $\mathrm{RMN}{ }^{13} \mathrm{C}-\mathrm{HBBD}, 20$ linhas espectrais e através da comparação deste com o espectro de RMN ${ }^{13} \mathrm{C}$ - DEPT $135^{\circ}$ foi possível caracterizar o padrão de hidrogenação relativo a cada átomo de carbono. Verificouse, então, que o composto $\mathbf{1}$ apresentava quatro sinais correspondentes a átomos de carbono não hidrogenados, seis sinais relativos a átomos de carbono metínicos, seis sinais correspondentes a átomos de carbono metilênicos e quatro sinais referentes a átomos de carbono metílicos. Observou-se ainda quatro sinais em $\delta 109,79 ; \delta 122,68 ; \delta$ 140,49 e $\delta 150,11$, característicos de átomos de carbono de anel furânico. Alguns dados foram confirmados pelo espectro de correlação heteronuclear HMQC (Tabela 1). Com base nestes dados espectrais, na determinação estrutural das substâncias isoladas (2 e 3) e em dados relatados na literatura (Mendes, 1987), foi possível concluir que a substância 1 tratava-se do diterpeno furânico vouacapano (1), sintetizado a partir do ácido vinhaticóico (Mendes, 1987) e identificado em Dipterix odorata por CGAR-EM (Godoy et al., 1989), através da co-injeção, mas inédito como produto natural.

Os diterpenos 6- $\alpha$-acetoxivouacapano (2) e 6- $\alpha$-hidroxivouacapano (3) foram identificadas pela comparação dos dados espectrais obtidos, com os da literatura (Mendes; Silveira, 1994) e (Arriaga et al., 2000), respectivamente.

O diterpeno 2, sólido branco amorfo com ponto de fusão na faixa de $122,3-124,3^{\circ} \mathrm{C}$, apresentou rotação específica $[\alpha]_{\mathrm{D}}{ }^{25}=+121,81$ (c 22,0, $\mathrm{CHCl}_{3}$ ). O espectro de $\mathrm{RMN}^{13} \mathrm{C}$ - HBBD (125 MHz, $\mathrm{CDCl}_{3}$ ) mostrou 22 linhas espectrais e através da comparação deste espectro com o espectro DEPT $135^{\circ}$, foi possível constatar que o diterpeno (2) continha 5 sinais correspondentes a átomos de carbono não hidrogenados, 7 sinais correspondentes a átomos de carbono metínicos, 5 sinais relativos a átomos de carbono metilênicos e 5 sinais correspondentes a átomos de carbono metílicos. Observou-se ainda dois sinais em $\delta 73,06$ e $\delta 170,83$, que foram atribuídos a um átomo de carbono carbinólico e a um átomo de carbono carbonílico, respectivamente. Foram observados quatro sinais em $\delta$ 109,$92 ; 122,62 ; 140,97 ; 149,77$, característicos de átomos de carbono de anel furânico. No espectro de $\mathrm{RMN}^{1} \mathrm{H}$ (500 $\mathrm{MHz}, \mathrm{CDCl}_{3}$ ) desta substância também foram observados os sinais correspondentes aos átomos de hidrogênio de três grupamentos metila ligados a átomos de carbono não hidrogenados em $\delta 0,94 ; 0,98$ e 1,08, além de outro sinal correspondente aos átomos de hidrogênio de uma metila ligada a átomo de carbono monoidrogenado em $\delta$ $1,00(\mathrm{~d}, J=7 \mathrm{~Hz})$. Foram observados dois dubletos em $\delta$ 6,18 e 7,22 $(J=1,8 \mathrm{~Hz})$, indicando se tratar de um anel furânico dissubstituído. Este mesmo espectro apresentou um triplo dubleto em $\delta 5,16(J=11,2$ e $5,3 \mathrm{~Hz})$ referente ao átomo de hidrogênio carbinólico. De acordo com estes dados foi possível caracterizar o diterpeno como $6-\alpha-$ acetoxivouacapano (2).

$\mathrm{O}$ espectro de $\mathrm{RMN}^{13} \mathrm{C}-\mathrm{HBBD}(125 \mathrm{MHz}$, $\mathrm{CDCl}_{3}$ ) do composto 3 apresentou vinte linhas espectrais, sendo 16 sinais correspondentes a átomos de carbono saturados e quatro sinais referentes a átomos de carbono insaturados, correspondentes aos átomos de carbono de um anel furânico. O espectro de $\mathrm{RMN}^{1} \mathrm{H}(500 \mathrm{MHz}$, $\mathrm{CDCl}_{3}$ ) mostrou além dos sinais correspondentes aos átomos de hidrogênio de quatro grupamentos metila e dos sinais correspondentes aos dois átomos de hidrogênio furânicos, um triplo dubleto em $\delta 3,99(J=10,8$ e 4,2 Hz), referente a um átomo de hidrogênio carbinólico. Desta forma, foi possível caracterizar a referida substância como 6- $\alpha$-hidroxivouacapano (3).

O óleo essencial obtido por hidrodestilação dos frutos de P. polygalaeflorus apresentou constituição semelhante à descrita na literatura (Campos et al., 1994b), apresentando o $\beta$-cariofileno como constituinte majoritário.

Tendo como objetivo a busca de novos produtos naturais que possam ser utilizados como larvicidas naturais, inicialmente foram testados sobre as larvas de A. aegypti, o extrato hexânico (EH) que mostrou $\mathrm{CL}_{50}$ igual a $23,99 \pm 0,75 \mu \mathrm{g} / \mathrm{mL}$, o extrato metanólico (EM) que não apresentou atividade, e o óleo essencial (OE) dos frutos de $P$. polygalaeflorus, cuja $\mathrm{CL}_{50}$ foi $134,90 \pm 0,25$ $\mu \mathrm{g} / \mathrm{mL}$. O 6- $\alpha$-acetoxivouocapano (2) isolado do extrato metanólico também foi testado e mostrou $\mathrm{CL}_{50}$ igual a $186,21 \pm 1,17 \mu \mathrm{g} / \mathrm{mL}$. O percentual de larvas mortas em diferentes concentrações permitiu determinar os valores de $\mathrm{CL}_{50}$ (Tabela 2).

Dentre as amostras analisadas foi possível constatar que o extrato hexânico, fração de menor polaridade dos frutos de $P$. polygalaeflorus, apresentou a 
Tabela 1. Dados de $\mathrm{RMN}{ }^{13} \mathrm{C}\left(125 \mathrm{MHz}, \mathrm{CHCl}_{3}\right), \mathrm{RMN}{ }^{1} \mathrm{H}\left(500 \mathrm{MHz}, \mathrm{CHCl}_{3}\right)$ e correlações ${ }^{1} \mathrm{H}-{ }^{13} \mathrm{C}\left(\mathrm{HMQC}-{ }^{1} J_{\mathrm{CH}}\right)$ de 1.

\begin{tabular}{|c|c|c|}
\hline & & HMQC \\
\hline $\mathrm{C}$ & $\delta \mathrm{C}$ & $\delta \mathrm{H}, J(\mathrm{~Hz})$ \\
\hline 4 & 33,41 & - \\
\hline 10 & 37,59 & - \\
\hline 13 & 122,68 & - \\
\hline 12 & 150,11 & - \\
\hline \multicolumn{3}{|l|}{$\mathbf{C H}$} \\
\hline 14 & 31,75 & $2,62(\mathrm{~m})$ \\
\hline 8 & 35,93 & \\
\hline 9 & 45,81 & \\
\hline 5 & 55,38 & \\
\hline 15 & 109,79 & $6,19(\mathrm{~d}, J=1,8)$ \\
\hline 16 & 140,49 & $7,23(\mathrm{~d}, J=1,8)$ \\
\hline \multicolumn{3}{|l|}{$\mathrm{CH}_{2}$} \\
\hline 2 & 18,97 & \\
\hline 6 & 21,77 & \\
\hline 11 & 22,42 & \\
\hline 7 & 31,47 & \\
\hline 1 & 39,92 & \\
\hline 3 & 42,17 & \\
\hline \multicolumn{3}{|l|}{$\mathrm{CH}_{3}$} \\
\hline 20 & 14,69 & $0,91(\mathrm{~s})$ \\
\hline 17 & 17,78 & $0,99(\mathrm{~d}, J=7,0)$ \\
\hline 18 & 22,46 & $0,88(\mathrm{~s})$ \\
\hline 19 & 33,91 & $0,91(\mathrm{~s})$ \\
\hline
\end{tabular}

Tabela 2. Valores de percentual de mortalidade das larvas de Aedes aegypti causadas pelos extratos, diterpeno 2 e óleo essencial de Pterodon polyglaeflorus.

\begin{tabular}{ccccc}
\hline Conc. $(\mu \mathrm{g} / \mathrm{mL})$ & EH & EM & 2 & OE \\
\hline 500 & 100 & 0 & 100 & 100 \\
250 & 100 & 0 & 65 & 99 \\
100 & 100 & 0 & 22 & 11 \\
50 & 100 & 0 & 20 & $\mathrm{nt}$ \\
25 & 53 & 0 & $\mathrm{nt}$ & $\mathrm{nt}$ \\
12,5 & 19 & 0 & $\mathrm{nt}$ & $\mathrm{nt}$ \\
\hline
\end{tabular}

$\mathrm{nt}=$ não testada

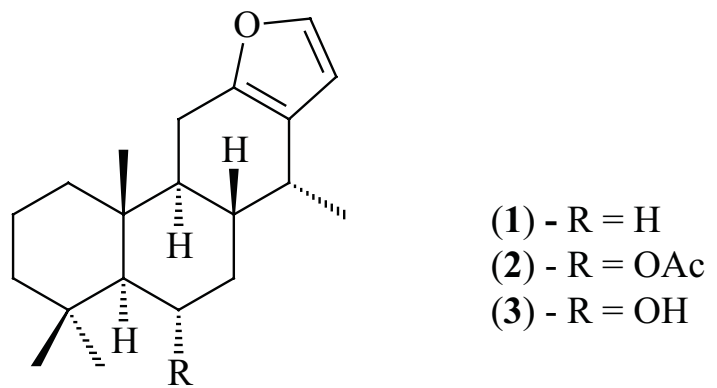

Figura 1. Estruturas dos diterpenos isolados de Pterodon polygalaeflorus 
melhor atividade frente às larvas de $A$. aegypti, com um valor de $\mathrm{CL}_{50}$ igual a $23,99 \pm 0,75 \mu \mathrm{g} / \mathrm{mL}$. De acordo com a literatura (Cheng et al., 2003), substâncias com valores de $\mathrm{CL}_{50}$ menores que $100 \mu \mathrm{g} / \mathrm{mL}$ são considerados bons agentes larvicidas.

\section{CONCLUSÃO}

Os resultados sugerem que o extrato hexânico dos frutos de $P$. polygalaeflorus é um promissor agente larvicida sobre $A$. aegypti.

\section{AGRADECIMENTOS}

Os autores agradecem ao CNPq, CAPES, FUNCAP e PRONEX pelo suporte financeiro e ao NUEND pela concessão das larvas.

\section{REFERÊNCIAS}

Arriaga AMC, Castro MAB, Silveira ER, Braz-Filho R 2000. Further diterpenoids isolated from Pterodon polygalaeflorus. J Braz Chem Soc 11: 187-190.

Barbosa-Filho JM, Medeiros KCP, Diniz MFFM, Batista LM, Athayde-Filho PF, Silva MS, Cunha EVL, Almeida JRGS, Quintans-Júnior LJ 2006. Natural products inhibitors of the enzyme acetylcholinesterase. Rev Bras Farmacogn 16: 258-285.

Campos AM, Silveira ER, Braz-Filho R, Teixeira TC 1994a. Diterpenoids from Pterodon polygalaeflorus. Phytochemistry 36: 403-406.

Campos AM, Alencar JW, Matos FJA, Machado MIL, Craveiro AA, Teixeira TC 1994b. Volatile constituents from fruits of Pterodon polygalaeflorus. J Essent Oil Res 6: 517-518.

Cheng SS, Chang HT, Chang ST, Tsai KH, Chen WJ 2003. Bioactivity of selected plant essential oils against the yellow fever mosquito Aedes aegypti larvae. Bioresource Technol 89: 99-102.

Demuner AJ, Barbosa LCA, Veloso DP, Alves DLF, Howarth WO 1996. Structure and plant growth regulatory activity of new diterpenes from Pterodon polygalaeflorus. J Nat Prod 59: 770-772.

Falcão HS, Lima IO, Santos VL, Dantas HF, Diniz MFFM, Barbosa-Filho JM, Batista LM 2005. Review of the plants with anti-inflammatory activity studied in Brazil. Rev Bras Farmacogn 15: 381-391.

Gadelha DP, Toda AT 1985. Biologia e comportamento do Aedes aegypti. Rev Bras Malariol Doenças Trop 37: 29-36.

Godoy LO, Lima DB, Pinto AC, Aquino-Neto, FR 1989. Diterpenoids from Dipterix odorata. Phytochemistry 28: 642-644.

Lima MRF, Ximenes CPA, Luna JS, Sant'Ana AEG 2006. The antibiotic activity of some Brazilian medicinal plants. Rev Bras Farmacogn 16: 300-306.

Lorenzi H 1998. Árvores brasileiras manual de identificação e cultivo de plantas arbóreas nativas do Brasil, Lisboa: Instituto Plantarun.

Mendes FNP, Silveira ER 1994. Fatty acids, sesqui- and diterpenoids from seeds of Dipteryx odorata.
Phytochemistry 35: 1499-1503.

Mendes FNP 1987. Contribuição ao estudo químico de plantas do Nordeste Brasileiro: Dipteryx lacunifera Ducke. Fortaleza, 134p. Dissertação de Mestrado - Departamento de Química Orgânica e Inorgânica, Universidade Federal do Ceará.

Oliveira MF, Lemos TLG, Mattos MC, Segundo TA, Santiago GMP, Braz-Filho R 2002. New enamines derivatived of lapachol and bilogical activity. An Acad Bras Cienc 74: 211-321.

Santiago GMP, Viana FA, Pessoa, ODL, Santos RP, Pouliquen YBM, Arriaga AMC, Andrade-Neto M, Braz-Filho R 2005. Avaliação da atividade larvicida de saponinas triperpênicas isoladas de Pentaclethra macroloba (Willd.) Kuntze (Fabacea) e Cordia piauhiensis Fresen (Boraginaceae) sobre Aedes aegypti. Rev Bras Farmacogn 15: 187-190.

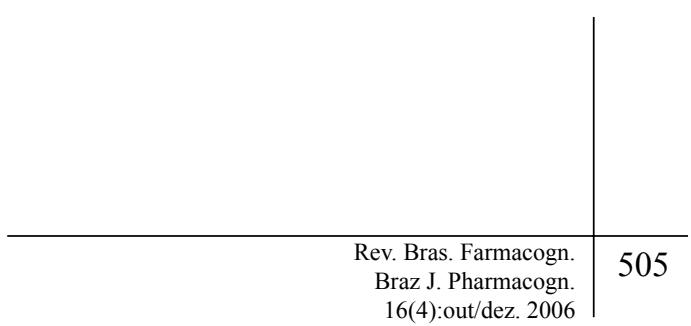

